\section{Asociación de un índice de estilos de vida saludable con factores de riesgo cardiovascular en población chilena}

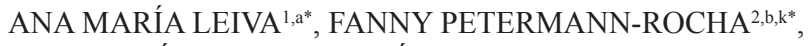 \\ MARÍA ADELA MARTÍNEZ-SANGUINETTI ${ }^{3, \mathrm{c}^{*} \text {, }}$ \\ CLAUDIA TRONCOSO-PANTOJA ${ }^{4, \mathrm{~d}}$, YENY CONCHA ${ }^{5,6, \mathrm{e}}$, \\ ALEX GARRIDO-MÉNDEZ7,8,f XIMENA DÍAZ-MARTÍNEZ ${ }^{9, \mathrm{~g}}$, \\ FABIÁN LANUZA-RILLING ${ }^{10, \mathrm{~b}}$, NATALIA ULLOA ${ }^{11, \mathrm{~h}}$, \\ MIQUEL MARTORELL ${ }^{12, \mathrm{i}}$, CRISTIAN ÁLVAREZ ${ }^{13, j}$, \\ CARLOS CELIS-MORALES ${ }^{14,15, \mathrm{k}}$, en representación del \\ Grupo de Investigación ELHOC
}

\section{Association of a healthy lifestyle index with cardiovascular risk factors in Chileans adults}

Background: Healthy lifestyles are associated with a better metabolic and cardiovascular health profile. Aim: To investigate the association between a lifestyle score and cardiovascular risk in Chilean adults. Material and Methods: A healthy lifestyle score was derived for 2,774 participants in the Chilean National Health Survey 2009-2010 and based on seven modifiable behaviors (salt intake, fruit and vegetable intake, alcohol consumption, sleep duration, smoking, physical activity and sedentary behaviors). A high score represented a healthier lifestyle whereas a low score represents an unhealthy lifestyle. The association between the lifestyle score and cardiovascular risk factors (obesity, hypertension, diabetes, dyslipidemia and metabolic syndrome), was explored using logistic regression models. Results: One quartile increment in the healthy lifestyle score was associated with a lower risk for obesity (Odds ratio (OR): 0.82 [95\% confidence intervals (CI): 0.75 to 0.90], $p<0.01$ ), central obesity (OR: 0.88 [95\% CI: 0.81 to 0.96], $p<0.01$ ), diabetes (OR: 0.84 [95\% CI: 0.75 to 0.95], $p<0.04$ ) and dyslipidemia (OR: 0.90 [95\% CI: 0.83 to 0.98$], p=0.01$ ). These results were independent of major confounding factors. Conclusions: The adherence to a healthy lifestyle is associated with lower cardiovascular risk.

(Rev Med Chile 2018; 146: 1405-1414)

Key words: Cardiovascular Diseases; Diabetes; Diet; Lifestyle; Obesity.
'Instituto de Anatomía, Histología y Patología, Facultad de Medicina, Universidad Austral de Chile. Valdivia, Chile

${ }^{2}$ Institute of Health and Wellbeing, University of Glasgow, Glasgow, United Kingdom.

${ }^{3}$ Instituto de Farmacia, Facultad de Ciencias, Universidad Austral de Chile, Valdivia, Chile. ${ }^{4}$ CIEDE-UCSC, Facultad de Medicina, Universidad Católica de la Santísima Concepción. Concepción, Chile. ${ }^{5}$ Escuela de Kinesiología, Facultad de Salud,

Universidad Santo Tomás, Chile.

${ }^{6}$ Universidad Tecnológica de Chile, INACAP, Chile. ${ }^{7}$ Escuela de Educación Física, Universidad San

Sebastián, Concepción, Chile.

${ }^{8}$ Departamento de Ciencias del Deporte y

Acondicionamiento Físico, Facultad de Educación, Universidad Católica de la Santísima Concepción. Concepción, Chile.

${ }^{9}$ Grupo de Investigación en Calidad de Vida, Departamento de Ciencias de la Educación, Facultad de Educación y Humanidades, Universidad del Biobío. Chillán, Chile.

${ }^{10}$ Departamento de Pediatría y Cirugía Infantil, Facultad de Medicina, Universidad de La Frontera, Temuco, Chile. ${ }^{11}$ Centro de Vida Saludable, Universidad de Concepción. Concepción, Chile.

${ }^{12}$ Departamento de Nutrición y Dietética. Facultad de Farmacia, Universidad de Concepción. Concepción, Chile.

${ }^{13} \mathrm{Grupo}$ de investigación de área prioritaria Calidad de Vida y Bienestar Humano. Departamento de Ciencias de la Actividad Física, Universidad de Los Lagos,

Osorno, Chile.

${ }^{14}$ BHF Glasgow Cardiovascular Research Centre, Institute of Cardiovascular and Medical Science, University of Glasgow, Glasgow, United Kingdom. ${ }^{15}$ Centro de Investigación en Fisiología del EjercicioCIFE, Universidad Mayor. Santiago, Chile.

aProfesora de Biología y Química. MSc. Neurociencias y Salud Mental.

${ }^{\text {b}}$ Nutricionista. MSc Nutrición Humana.

'Bioquímica. MSc Nutrición y Dietética.

dMSc Salud Pública basada en la evidencia; MSc Gerontología.

eMSc Ciencias de la Actividad Física.

${ }^{\mathrm{f}}$ Dr Actividad Física, Educación Física y Deporte.

gprofesora de Educación Física, MSc Educación Física.

hDr Ciencias Biológicas; MSc Bioquímica clínica.

'Dr Nutrición Humana; MSc Nutrigenómica y Nutrición personalizada.

iDoctor ${ }^{\circ}$.

${ }^{k}$ Dr Ciencias Cardiovasculares y Biomédicas.

${ }^{*} \mathrm{AML}, \mathrm{FP}$ y MAM contribuyeron igualmente a este trabajo y son consideradas como primer autor compartido.

Trabajo no recibió financiamiento.

Los autores declaran no tener conflictos de interés. Recibido 26 de julio de 2018, aceptado el 16 de noviembre de 2018

Correspondencia a:

Carlos Celis-Morales

BHF Glasgow Cardiovascular Research Centre, Institute of Cardiovascular and Medical Sciences, College of Medical, Veterinary and Life Sciences, University of Glasgow, Glasgow. G12 8TA, United Kingdom.

Tel: 044 (0) 1413304201; Fax: 044 (0) 1413305481 address: Carlos.Celis@glasgow.ac.uk 
E crecimiento económico, la urbanización y la globalización han modificado profundamente el estilo de vida del siglo XXI, lo que ha afectado de forma significativa la salud de la población a nivel mundial. El cambio en los perfiles epidemiológicos de las poblaciones se ha reflejado en el incremento ponderal de la obesidad y de las enfermedades cardiovasculares (ECVs), que son la principal causa de mortalidad en América Latina y en Chile ${ }^{1}$. Se estima que al menos $30 \%$ de las defunciones registradas en el mundo el año 2012 fueron a causa de ECVs².

Diversos estudios han establecido el rol preponderante del estilo de vida en el desarrollo de factores de riesgo cardiovascular y $\mathrm{ECVs}^{3-5}$, existiendo consenso que una de las maneras más eficaces de prevenir y/o disminuir la prevalencia de estas patologías es fomentar hábitos de vida saludable con un enfoque integral, centrado en la alimentación saludable, la práctica de actividad física $(\mathrm{AF})$, prevención del tabaquismo, consumo de alcohol, drogas y la promoción de la salud mental del individuo ${ }^{6}$. Las personas que exhiben un mayor número de estos hábitos de vida saludable presentan una tasa de mortalidad significativamente menor que aquellas que poseen factores de riesgos conductuales ${ }^{7,8}$.

Por otra parte, estudios internacionales han demostrado resultados positivos al utilizar un índice de estilo de vida saludable (IES) como un indicador útil para la prevención de ECVs ${ }^{9,10}$. Este tipo de indicador reúne información en relación con las conductas de los individuos, como las descritas con anterioridad. Actualmente, en Chile no se ha definido un índice que permita establecer este tipo de asociación; sin embargo, la Encuesta Nacional de Salud (ENS) 2009-2010 $0^{11}$ proporciona resultados que permitirían crearlo e identificar de manera global el riesgo de desarrollar ECVs. Por lo tanto, el objetivo de este trabajo fue investigar la asociación de un índice de estilo de vida saludable con factores de riesgo cardiovascular en población chilena.

\section{Materiales y Métodos}

\section{Diseño del estudio}

La muestra seleccionada comprende a los participantes de la ENS 2009-2010 de Chile, desarrollada entre octubre del año 2009 y septiembre del año 2010 ${ }^{11}$. La ENS 2009-2010 correspondió a un estudio de prevalencia realizado en hogares a partir de una muestra nacional, probabilística, estratificada y multietápica de 5.412 personas mayores de 15 años con representatividad nacional, regional, y área urbana/rural. En este estudio se incluyó un total de 2.774 participantes (58,3\% mujeres), que tenían información disponible en relación con sus estilos de vida. El protocolo de estudio fue aprobado por el Comité de Ética de la Escuela de Medicina de la Pontificia Universidad Católica de Chile. Todos los participantes firmaron un consentimiento informado ${ }^{11}$.

\section{Mediciones metabólicas y antropométricas}

Entre los factores de riesgo cardiovascular evaluados se encuentra diabetes mellitus tipo 2 (DMT2), colesterol total, colesterol HDL, triglicéridos, síndrome metabólico (SM), hipertensión arterial (HTA), obesidad y obesidad central. Las muestras de sangre de los participantes fueron obtenidas en ayuno por una enfermera entrenada siguiendo protocolos estandarizados a nivel nacional ${ }^{11}$. La glicemia basal fue medida con métodos estandarizados y previamente descritos en la ENS 2009-2010 ${ }^{11}$. Para la detección de DMT2 se consideró una glicemia en ayuno $\geq 126 \mathrm{mg} / \mathrm{dl}$ o diagnóstico previo de DMT2 ${ }^{12}$. El control metabólico de DMT2 se realizó con la medición de hemoglobina glicosilada ( $\mathrm{HbAc1})$. Colesterol y triglicéridos altos fueron definidos con un valor de $>200 \mathrm{mg} /$ dl y $>150 \mathrm{mg} / \mathrm{dl}$, respectivamente. Valores $<40$ $\mathrm{mg} / \mathrm{dl}$ en hombres $\mathrm{y}<50 \mathrm{mg} / \mathrm{dl}$ en mujeres fueron asociados a colesterol HDL bajo. Se consideró SM a la presencia de 3 o más de un total de 5 criterios definidos en ATP III Update 2004 ${ }^{13}$. La HTA fue definida a través de tres mediciones con valores $\geq 140 / 90 \mathrm{mmHg}$ o el auto-reporte de tratamiento farmacológico para HTA.

El peso corporal fue medido a través de una balanza (Tanita HD-313 ${ }^{\circledR}$ ) con una precisión de 0,1 $\mathrm{kg}$. La talla fue medida en centímetros a través de un tallímetro con cartabón incorporado. Con estos dos datos se obtuvo el estado nutricional a través del cálculo del Índice de Masa Corporal (IMC, kg/ $\mathrm{m}^{2}$ ), cuyos valores se clasificaron acorde al reporte del año 2000 de la Organización Mundial de la Salud (OMS): bajo peso: $<18,5 \mathrm{~kg} / \mathrm{m}^{2}$; normo peso: $18,5-24,9 \mathrm{~kg} / \mathrm{m}^{2}$; sobrepeso: $25,0-29,9 \mathrm{~kg} / \mathrm{m}^{2}$ y obesidad: $\geq 30,0 \mathrm{~kg} / \mathrm{m}^{2(14)}$. La obesidad central fue definida como un perímetro de cintura (PC) 
$\geq 80 \mathrm{~cm}$ para mujeres $\mathrm{y} \geq 90 \mathrm{~cm}$ para hombres, según recomendación internacional utilizada en el consenso 2014 del Ministerio de Salud para la prevención de $\mathrm{ECV}^{15}$

\section{Variables sociodemográficas y de estilo de vida}

Las variables sociodemográficas (edad, sexo, nivel educacional, ingreso económico, zona geográfica) y datos asociados con estilos de vida, como el tabaquismo, consumo de alcohol, consumo de frutas y verduras, se obtuvieron mediante la aplicación de cuestionarios validados en la ENS 2009-2010 ${ }^{11}$. El consumo de sal se determinó mediante análisis de excreción de sodio en orina de 24 horas, a través de la fórmula de Tanaka $2002^{16}$.

\section{Clasificación de actividad física}

Los niveles de AF, el tiempo destinado a las actividades de transporte y las actividades de intensidad moderada o vigorosa de la población, fueron determinados con el cuestionario "Global Physical Activity Questionnaire” (GPAQ v2) ${ }^{17}$, el cual ha sido validado internacionalmente ${ }^{18} \mathrm{y}$ en población latina ${ }^{19}$. La AF total es presentada como la suma del tiempo reportado en actividades de transporte, de intensidad moderada y vigorosa tanto en el trabajo como en el tiempo libre. Esta variable fue expresada en MET/minutos/semana para considerar las diferencias en gasto energético asociada a cada una de las actividades. Se consideró como punto de corte para inactividad física un gasto energético menor a $600 \mathrm{METs} /$ minutos/ semana $^{17,20,21}$. Los niveles de sedentarismo fueron determinados mediante el auto-reporte de tiempo destinado a actividades que involucren estar sentado o reclinado durante el tiempo libre o de trabajo mediante el mismo cuestionario ${ }^{17}$.

\section{Cálculo y cuartiles de índice de estilo de vida saludable}

Para la creación de un IES, se utilizaron 7 conductas propuestas por la OMS y relacionadas a estilos de vida saludable ${ }^{22}$, y que además se encontraban disponible en la ENS 2009-2010, incluyendo, 1) consumo de 5 porciones de frutas y verduras al día; 2) consumo de sal $<8 \mathrm{~g}$ al día; 3 ) puntaje test AUDIT de consumo de alcohol $<8$ (método simple de screening del consumo excesivo de alcohol creado por la $\mathrm{OMS}^{23}$ ); 4) horas de sueño entre 7-9 h al día; 5) nunca haber fumado; 6) físicamente activo ( $>150 \mathrm{~min}$ a la semana de AF moderada); y 7) tiempo sedente $<4 \mathrm{~h}$ al día ${ }^{24}$. A los individuos que cumplían con estas recomendaciones se les asignaba 1 punto por cada conducta saludable que cumplían, mientras que aquellos que no cumplían con estas recomendaciones se le asignaban un valor cero. El puntaje máximo fue 7 puntos, que indicaba que cumplía con todas las conductas relacionadas con un estilo de vida saludable, y 0 que representa a individuos que no cumplían con ninguno de las recomendaciones. Para propósitos de este estudio, se clasificó el puntaje del IES en 4 categorías o cuartiles: no saludable (puntaje 0 a 2; cuartil 1), poco saludable (puntaje: 3; cuartil 2), medianamente saludable (puntaje: 4; cuartil 3), y saludable (puntaje: 5 a 7; cuartil 4).

\section{Análisis estadístico}

Los datos de caracterización de la población estudiada son presentados como promedio y desviación estándar (DE) para variables continuas, y como porcentaje para variables categóricas.

Para evaluar diferencias en variables metabólicas y antropométricas entre los grupos de IES, se realizó un análisis de tendencia mediante regresión lineal, donde se reportó el coeficiente beta para la tendencia y su respectivo valor p, es decir, en cuánto se modifica en promedio la variable de salida (variables antropométricas o metabólicas) equivalente a desplazarse a la siguiente categoría de IES.

Análisis de regresión logística fueron aplicados para estimar la probabilidad de tener un nivel alterado de factores de riesgo cardiovasculares, donde se presenta el odds ratio (OR) y sus respectivos intervalos de confianza (95\% IC) para cada categoría del IES en comparación al grupo de referencia (grupo 1 con índice de estilo de vida no saludable). También se estimó el OR de tendencia para determinar en cuánto incrementa la probabilidad de tener estos factores de riesgo en la medida que el IES aumenta en una categoría. Todos los análisis fueron ajustados con variables de confusión incluyendo edad, sexo, nivel educacional y zona geográfica de residencia (rural/urbana).

Para todos los análisis se utilizó el módulo de análisis de muestras complejas del programa STATA SE v14 y todos los resultados fueron estimados utilizando muestras expandidas según la ENS 2009-2010 ${ }^{11}$. El nivel de significancia fue definido como $\mathrm{p}<0,05$. 


\section{Resultados}

La Tabla 1 muestra un resumen de las características generales de la población según cuartiles de IES, observándose que son las mujeres quienes presentan parámetros de salud cardiometabólica y de estilo de vida más saludables. Se identifican, además, diferencias entre los distintos grupos según su categorización. Las personas agrupadas en el cuartil 1 (menos saludable) concentraron los mayores niveles de sobrepeso/obesidad y obesidad central. También este grupo es el que concentró los menores niveles de AF en sus diferentes categorizaciones (total, de transporte, moderada $\mathrm{y}$ vigorosa), prevaleciendo la inactividad física $\mathrm{y}$ el tiempo sedente por sobre los otros grupos. En este contexto, es importante destacar que, aquellas personas que permanecen mayor tiempo sedentes,

Tabla 1. Características de la población según cuartiles de Índice de estilo de vida saludable

\begin{tabular}{|c|c|c|c|c|}
\hline \multirow[b]{2}{*}{ Variables } & \multicolumn{4}{|c|}{ Cuartiles Índice de Estilos de Vida saludable } \\
\hline & $\begin{array}{c}1 \\
\text { (No saludable) }\end{array}$ & $\begin{array}{c}2 \\
\text { (Poco } \\
\text { Saludable) }\end{array}$ & $\begin{array}{c}3 \\
\text { (Medianamente } \\
\text { Saludable) }\end{array}$ & $\begin{array}{c}4 \\
\text { (Saludable) }\end{array}$ \\
\hline $\begin{array}{l}\text { Sociodemográficas } \\
\text { Total (n) } \\
\text { Edad (años) } \\
\text { Sexo, mujeres (\%) }\end{array}$ & $\begin{array}{c}458 \\
46,5(19,6) \\
46,3\end{array}$ & $\begin{array}{c}778 \\
46,2(18,8) \\
56,3\end{array}$ & $\begin{array}{c}842 \\
4,3(18,3) \\
60,0\end{array}$ & $\begin{array}{c}696 \\
46,7(17,7) \\
66,5\end{array}$ \\
\hline $\begin{array}{l}\text { Nivel educacional (\%) } \\
\text { Básica ( }<8 \text { años) } \\
\text { Media (< } 12 \text { años) } \\
\text { Técnico o Universitaria }\end{array}$ & $\begin{array}{l}26,9 \\
50,6 \\
22,5\end{array}$ & $\begin{array}{l}27,7 \\
52,5 \\
19,8\end{array}$ & $\begin{array}{l}25,3 \\
56,4 \\
18,3\end{array}$ & $\begin{array}{l}26,0 \\
56,0 \\
18,0\end{array}$ \\
\hline $\begin{array}{l}\text { Nivel de ingreso (\%) } \\
\text { Bajo } \\
\text { Medio } \\
\text { Alto }\end{array}$ & $\begin{array}{l}54,9 \\
33,1 \\
12,0\end{array}$ & $\begin{array}{l}54,9 \\
32,9 \\
12,2\end{array}$ & $\begin{array}{l}55,9 \\
32,6 \\
11,5\end{array}$ & $\begin{array}{l}55,5 \\
33,3 \\
12,2\end{array}$ \\
\hline $\begin{array}{l}\text { Estado nutricional (\%) } \\
\text { Bajo peso } \\
\text { Normopeso } \\
\text { Sobrepeso } \\
\text { Obesidad } \\
\text { Circunferencia de cintura (cm) } \\
\text { Obesidad central (\%) }\end{array}$ & $\begin{array}{c}1,1 \\
24,2 \\
40,5 \\
34,2 \\
98,6(13,1) \\
75,9\end{array}$ & $\begin{array}{c}2,1 \\
28,2 \\
37,3 \\
32,4 \\
97,0(12,8) \\
70,9\end{array}$ & $\begin{array}{c}1,0 \\
31,6 \\
40,5 \\
26,9 \\
95,3(11,4) \\
71,0\end{array}$ & $\begin{array}{c}2,2 \\
32,9 \\
38,3 \\
26,6 \\
95,1(11,7) \\
69,5\end{array}$ \\
\hline $\begin{array}{l}\text { Estilos de vida } \\
\text { Actividad física total (MET/h/semana) } \\
\text { Actividad física de transporte (min/día) } \\
\text { Actividad física moderada (min/día) } \\
\text { Actividad física vigorosa (min/día) } \\
\text { Prevalencia inactividad física (\%) } \\
\text { Tiempo sedente (h/día) } \\
\text { Consumo de frutas y verduras (g/día) } \\
\text { Consumo de sal (g/día) } \\
\text { Consumo de alcohol (g/día) }\end{array}$ & $\begin{array}{c}77,1(137,8) \\
30,7(71,3) \\
57,0(126,6) \\
38,7(112,7) \\
54,8 \\
5,35(2,9) \\
175,1(105,7) \\
10,7(3,4) \\
68,8(72,3)\end{array}$ & $\begin{array}{c}118,6(152,6) \\
48,3(93,4) \\
99,0(147,6) \\
53,5(125,9) \\
30,1 \\
3,82(2,7) \\
207,3(125,3) \\
10,1(2,6) \\
53,1(60,1)\end{array}$ & $\begin{array}{c}134,0(150,3) \\
54,8(87,9) \\
110,9(150,8) \\
60,7(133,3) \\
16,3 \\
3,07(2,5) \\
213,9(135,6) \\
9,7(2,9) \\
43,6(59,3)\end{array}$ & $\begin{array}{c}137,6(135,2) \\
55,6(80,2) \\
127,8(150,6) \\
55,8(120,8) \\
5,9 \\
2,25(1,7) \\
252,2(162,6) \\
8,9(2,6) \\
34,0(42,0)\end{array}$ \\
\hline $\begin{array}{l}\text { Tabaquismo (\%) } \\
\text { Nunca } \\
\text { Ex-fumador } \\
\text { Fumador }\end{array}$ & $\begin{array}{l}10,7 \\
37,5 \\
51,8\end{array}$ & $\begin{array}{l}27,9 \\
28,3 \\
43,8\end{array}$ & $\begin{array}{l}46,2 \\
21,4 \\
32,4\end{array}$ & $\begin{array}{l}71,1 \\
11,8 \\
17,1\end{array}$ \\
\hline
\end{tabular}

Datos presentados como media y su respectiva desviación estándar para variables continuas y cómo porcentaje para variables categóricas. 
concentran también otros hábitos no saludables como un mayor consumo de sal, alcohol y tabaco. No se observaron diferencias entre cuartiles de IES en cuanto a la edad, nivel educacional y nivel de ingreso económico.

$\mathrm{Al}$ analizar la asociación entre un IES y los marcadores de obesidad y metabólicos (Figuras 1 y 2), los resultados muestran que, por cada incremento en 1 cuartil de IES existe una tendencia significativa a disminuir en $1,96 \mathrm{~kg}$ el peso corporal; 0,41 $\mathrm{kg} / \mathrm{m}^{2}$ el IMC y $1,17 \mathrm{~cm}$ el perímetro de cintura (Figura 1), mientras que los marcadores metabólicos disminuyeron en 1,06 $\mathrm{mmHg}$ para presión arterial sistólica y en $0,10 \mathrm{mmol} / \mathrm{l}$ para triglicéridos (Figura 2). De igual manera, por cada 1 cuartil de IES existió una tendencia significativa a incrementar en $0,03 \mathrm{mmol} / \mathrm{l}$ el colesterol HDL (Figura 2). No se encontraron asociaciones estadísticamente significativas para glicemia, $\mathrm{HbAC1}$, presión arterial diastólica y colesterol total (Figura 2).

Finalmente, al evaluar la asociación entre el IES y los factores de riesgo cardiovascular (Figura 3), se observó que por cada 1 cuartil de incremento de este índice existió una tendencia a disminuir la obesidad (OR: 0,82 [95\% IC: 0,75 a 0,90], $\mathrm{p}<0,0001$ ), la obesidad central (OR: 0,88 [95\% IC: $0,81$ a 0,96$], \mathrm{p}=0,002$ ), la DMT2 (OR: 0,84 [95\% IC: 0,75 a 0,95$], p=0,004)$ y los triglicéridos elevados (OR: 0,90 [95\% IC: 0,83 a 0,98], p = 0,011). No se encontraron asociaciones estadísticamente significativas entre IES y SM, colesterol total, HDL reducido (Figura 3). Estos resultados fueron independientes de factores de confusión.

\section{Discusión}

Los principales hallazgos de este estudio confirman que en la medida que aumentan las conductas saludables disminuyen los factores de riesgo cardiovascular. Acorde a los datos de la última Encuesta Nacional de Salud 2017, en Chile un gran porcentaje de la población presenta una alta carga de riesgo cardiometabólico reflejado en la elevada prevalencia de exceso de peso corporal (74,2\% de la población), HTA $(27,6 \%)$ y DMT2 $(12,3 \%)^{25}$. En este contexto, los resultados obtenidos en este estudio cobran una gran relevancia considerando que, si los individuos modificaran su estilo de vida, es decir, se incrementase en 1 cuartil de IES, se obtendrían grandes beneficios

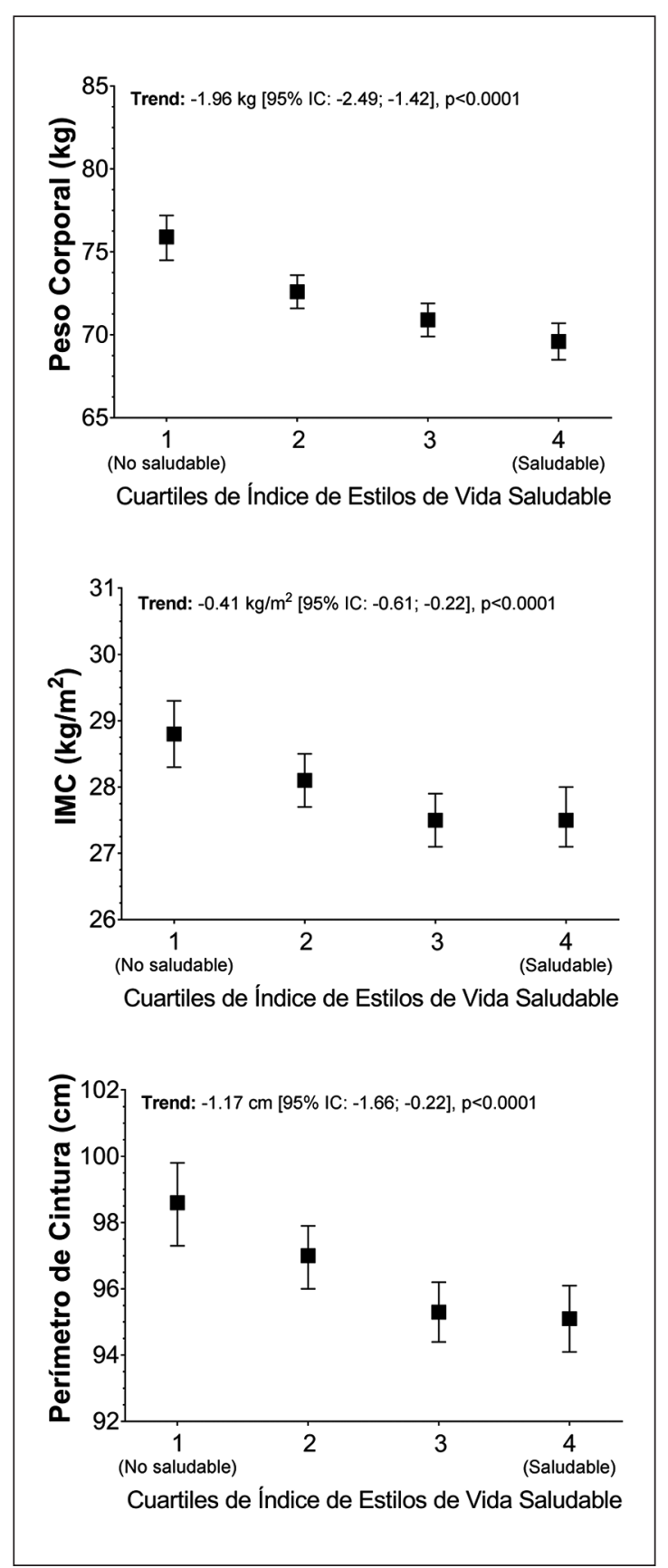

Figura 1. Asociación entre cuartiles de IES y marcadores de obesidad. Datos presentados como promedio y su respectivo $95 \%$ IC. El coeficiente beta para tendencia representa el incremento en la variable de salida de interés (variables de adiposidad) por cada 1 cuartil de aumento en el IES tomando como referencia el cuartil 1 (grupo con estilo de vida no saludable). Todos los análisis fueron ajustados con variables de confusión incluyendo edad, sexo, nivel educacional y zona geográfica de residencia (rural/urbana). 

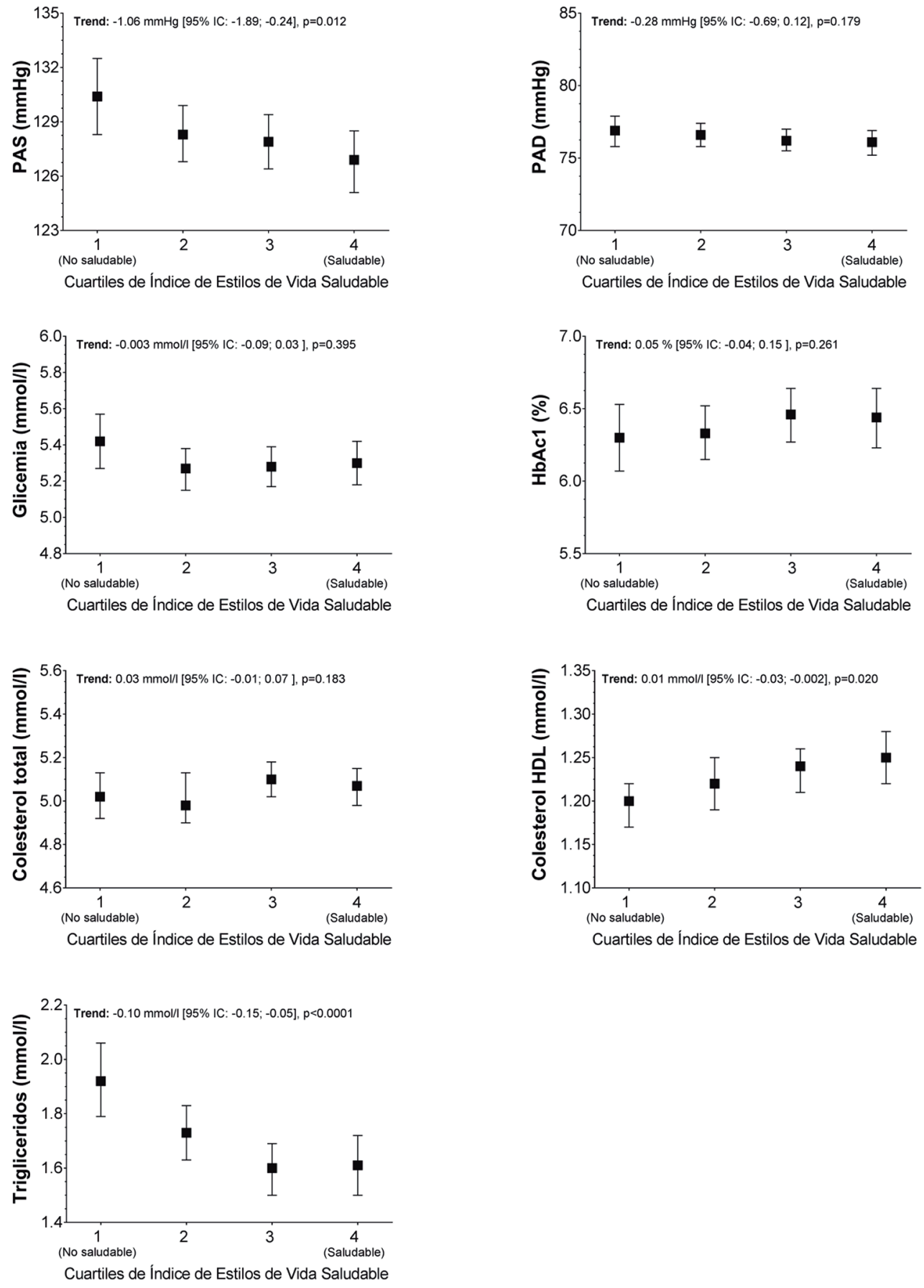

Figura 2. Asociación entre cuartiles de IES y marcadores metabólicos. Datos presentados como promedio y su respectivo $95 \%$ IC. El coeficiente beta para tendencia representa el incremento en la variable de salida de interés (marcador metabólico) por cada 1 cuartil de aumento en el IES tomando como referencia el cuartil 1 (grupo con estilo de vida no saludable). Todos los análisis fueron ajustados con variables de confusión incluyendo edad, sexo, nivel educacional, zona geográfica de residencia (rural/urbana) e IMC. 

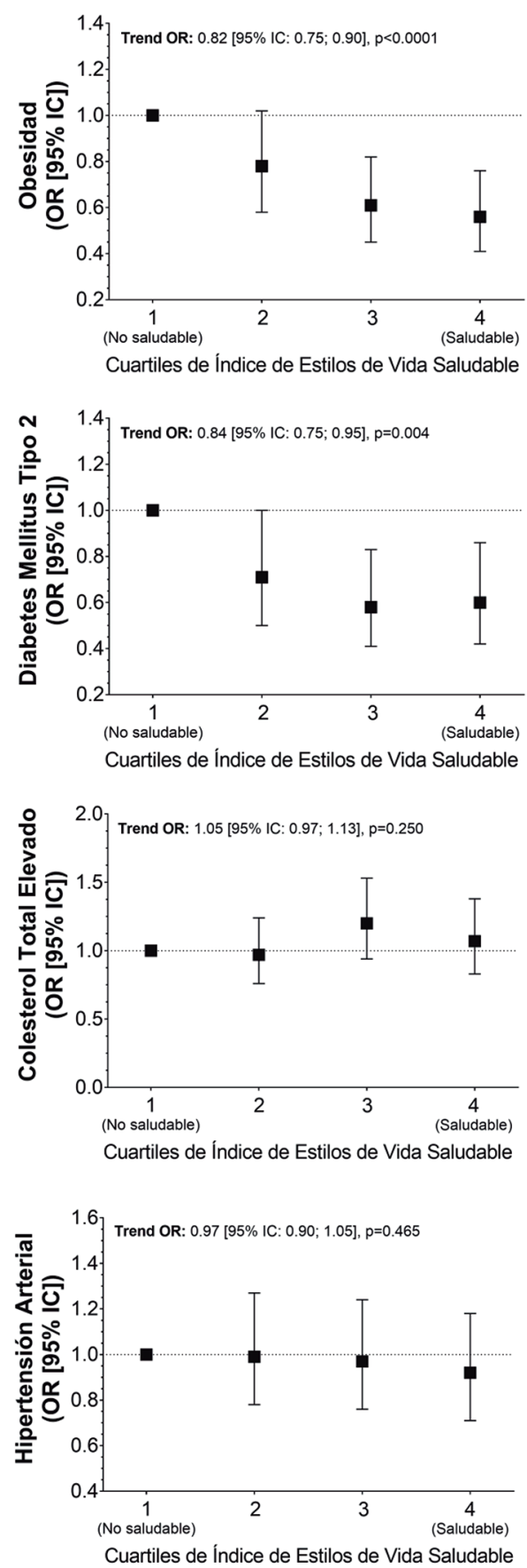
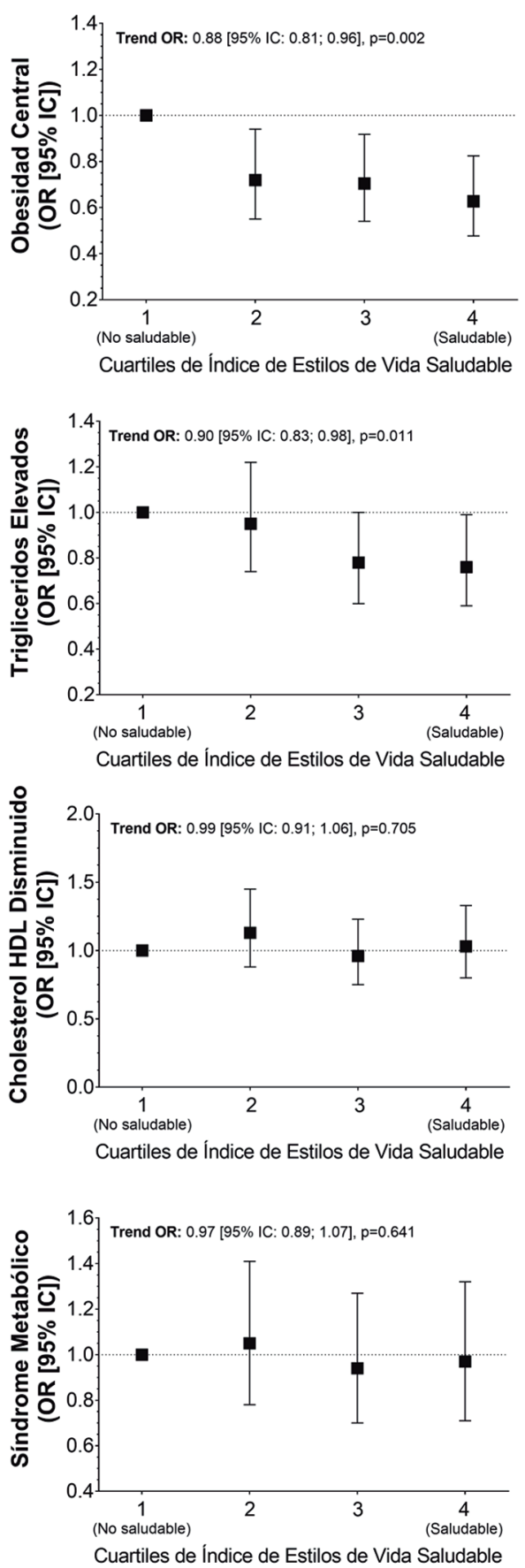

Figura 3. Asociación entre cuartiles de IES y factores de riesgo cardiovascular. Datos presentados como odds ratio (OR) y su respectivo $95 \%$ IC. El OR para tendencia representa la probabilidad de tener algún factor de riesgo cardiovascular alterado por cada un cuartil de aumento en el IES tomando como referencia al cuartil 1 (grupo con estilos de vida no saludable). Un OR bajo 1 indica una menor probabilidad de tener un factor de riesgo cardiovascular. Todos los análisis fueron ajustados con variables de confusión incluyendo edad, sexo, nivel educacional y zona geográfica de residencia (rural/urbana). *Marcadores de salud metabólica fueron ajustados de forma adicional por IMC. 
sobre la disminución de la obesidad, obesidad central, DMT2 y los triglicéridos elevados. En este sentido, un reciente estudio longitudinal realizado por la Universidad de Michigan, basado en una encuesta sobre salud aplicada a los estadounidenses mayores de 50 años, confirma la importancia de estos hallazgos, constatando que quienes exhibían un perfil de comportamiento más saludable, presentaban una esperanza de vida de siete años más y experimentaban un retraso en el inicio de la discapacidad de hasta seis años ${ }^{26}$.

Diversos estudios han respaldado la importancia de un estilo de vida saludable en la prevención de enfermedades cardiometabólicas ${ }^{27-29}$, una menor cantidad de estudios han analizado el efecto combinado de múltiples componentes del estilo de vida sobre la salud $\mathrm{d}^{30}$. A pesar que en este estudio no observó cambios significativos en el SM, Soto-Prieto $y$ cols. en un estudio en adultos puertorriqueños, evidenciaron que a través de la definición de un IES, y la corrección de múltiples comportamientos no saludables, se puede proporcionar una protección más fuerte contra el SM que la modificación de los componentes individuales ${ }^{31}$. Del Gobbo y cols. demostraron que patrones como la AF regular, el consumo moderado de alcohol y no fumar se asocian con un menor riesgo de insuficiencia cardíaca en adultos mayores estadounidenses ${ }^{32}$. De la misma manera, Chen y cols. identificaron en 5.349 personas de mediana y avanzada edad de Taiwán que, quienes presentaban un estilo de vida saludable (como un alto consumo de frutas, verduras, granos integrales, nueces, legumbres y realizar AF) presentaban un menor riesgo de desarrollar DMT2 $2^{33}$. Otro estudio desarrollado en 4 ciudad de Estados Unidos, por Liu y cols., indicó que la prevalencia del perfil de bajo riesgo aumenta a medida que el número de estilo de vida saludables incrementan y que mantener un comportamiento saludable durante la juventud está fuertemente asociado con un bajo riesgo de enfermedad cardiovascular en la mediana edad ${ }^{34}$.

Entre las fortalezas de este estudio se encuentran la generación de un IES junto con la utilización de datos provenientes de la ENS 2009-2010, encuesta que posee una muestra representativa de la población adulta chilena a nivel nacional. La utilización de un IES ofrece la posibilidad de capturar el efecto en conjunto de diferentes estilos de vida, que refleja en mayor medida la realidad de nuestras conductas en el día a día, en comparación a establecer asociaciones entre cada uno de los estilos de vida por separado. Además, se realizó la medición de variables cómo glicemia, colesterol total y presión arterial siguiendo protocolos estandarizados. Sin embargo, las variables asociadas al estilo de vida, como la práctica de AF, tiempo sedente e ingesta de alcohol, frutas y verduras, fueron auto-informadas y se ha demostrado previamente que podrían ser propensas a sesgo por este motivo ${ }^{35,36}$. Esto podría enmascarar la verdadera asociación entre el IES y factores de riesgo cardiovascular, por lo tanto, futuros estudios deberían considerar medidas objetivas tanto para la medición de la AF como para la ingesta dietética. Finalmente, como con todos los estudios observacionales, el uso de datos transversales no permite ninguna inferencia causal a partir de estos resultados.

\section{Conclusión}

La creación de un índice que reúne un conjunto de estilos de vida saludables (IES), permite identificar de manera global el riesgo cardiovascular en la población, confirmando los importantes beneficios que se podrían obtener si se tomase consciencia respecto a exhibir un estilo de vida saludable a lo largo del ciclo de vida. Para ello es necesario educar a la población desde la primera infancia, ya que, es en esta etapa donde se comienzan a adoptar los estilos de vida que exhibiremos en la vida adulta.

Agradecimientos: Se agradece de manera especial a todos los participantes de la ENS 20092010, al equipo profesional de la Escuela de Salud Pública, de la Facultad de Medicina de la Pontificia Universidad Católica de Chile, quienes desarrollaron y aplicaron la Encuesta Nacional de Salud y al Ministerio de Salud del Gobierno de Chile.

\section{Referencias}

1. Organization WH. Salud en las Americas+, Resumen: Panorama Regional y Perfiles de País. Washington DC. 2017; Available: http://iris.paho.org/xmlui/handle/123456789/34322.

2. Organization WH. Cardiovascular Diseases. Available: http://wwwwhoint/mediacentre/factsheets/fs317/es/. 2015. 
3. Anderson TJ. Importance of Optimization of Cardiovascular Risk Factors and Lifestyle Behaviours. Can J Cardiol 2017; 33 (10): 1221-2.

4. Ford ES, Bergmann MM, Boeing H, Li C, Capewell S. Healthy lifestyle behaviors and all-cause mortality among adults in the United States. Prev Med 2012; 55 (1): 23-7.

5. Loef M, Walach $\mathrm{H}$. The combined effects of healthy lifestyle behaviors on all cause mortality: a systematic review and meta-analysis. Prev Med 2012; 55 (3): 16370.

6. Atalah E. Epidemiología de la obesidad en Chile. Rev Med Clin Condes 2012; 23 (2): 117-23.

7. Tamakoshi A, Tamakoshi K, Lin Y, Yagyu K, Kikuchi S. Healthy lifestyle and preventable death: findings from the Japan Collaborative Cohort (JACC) Study. Prev Med 2009; 48 (5): 486-92.

8. Younus A, Aneni EC, Spatz ES, Osondu CU, Roberson L, Ogunmoroti O, et al. A Systematic Review of the Prevalence and Outcomes of Ideal Cardiovascular Health in US and Non-US Populations. Mayo Clin Proc 2016; 91 (5): 649-70.

9. Lucini D, Zanuso S, Blair S, Pagani M. A simple healthy lifestyle index as a proxy of wellness: a proof of concept. Acta Diabetol 2015; 52 (1): 81-9.

10. Díaz-Gutiérrez J, Ruiz-Canela M, Gea A, Fernández-Montero A, Martínez-González MA. Association Between a Healthy Lifestyle Score and the Risk of Cardiovascular Disease in the SUN Cohort. Rev Esp Cardiol (English ed). 2017.

11. MINSAL. Encuesta Nacional de Salud 2009-2010. Chile: Ministerio de Salud; 2010.

12. ADA. Report of the Expert Committee on the Diagnosis and Classification of Diabetes Mellitus. Diabetes Care. 1997; 20 (7): 1183-97.

13. Grundy SM, Cleeman JI, Merz CN, Brewer HB, Jr., Clark LT, Hunninghake DB, et al. Implications of recent clinical trials for the National Cholesterol Education Program Adult Treatment Panel III guidelines. Circulation. 2004; 110 (2): 227-39.

14. WHO. Obesity: Preventing and managing the Global Epidemic. 2000.

15. MINSAL. Enfoque de Riesgo para la Prevención de Enfermedades Cardiovasculares. Consenso 2014. Ministerio de Salud; Gobierno de Chile.

16. Tanaka T, Okamura T, Miura K, Kadowaki T, Ueshima $\mathrm{H}$, Nakagawa $\mathrm{H}$, et al. A simple method to estimate populational 24-h urinary sodium and potassium excretion using a casual urine specimen. J Hum Hypertens 2002; 16 (2): 97-103.

17. WHO. Global Physical Activity Questionnaire: GPAQ version 2.0. World Health Organization; 2009.

18. Bull FC, Maslin TS, Armstrong T. Global Physical Activity Questionnaire (GPAQ): Nine Country Reliability and Validity Study. J Phys Act Health 2009; 6 (6): 790 804.

19. Hoos T, Espinoza N, Marshall S, Arredondo EM. Validity of the Global Physical Activity Questionnaire (GPAQ) in Adult Latinas. J Phys Act Health 2012; 9 (5): 698-705.

20. Ainsworth BE, Haskell WL, Herrmann SD, Meckes N, Bassett DR, Jr., Tudor-Locke C, et al. 2011 Compendium of Physical Activities: A Second Update of Codes and MET Values. Med Sci Sports Exerc 2011; 43 (8): 1575-81.

21. WHO. Global recommendations on physical activity for health. World Health Organization; 2010.

22. WHO. Aumentar el consumo de frutas y verduras para reducir el riesgo de enfermedades no transmisibles. World Health Organization. Available: http://www.who. int/elena/titles/fruit_vegetables_ncds/es/.

23. WHO. AUDIT: Cuestionario de Identificación de los Transtornos debidos al Consumo de Alcohol. World Health Organization. 2001; Available: http://www.who. int/substance_abuse/activities/en/AUDITmanualSpanish.pdf.

24. Celis-Morales C, Salas C, Alduhishy A, Sanzana R, Martinez MA, Leiva A, et al. Socio-demographic patterns of physical activity and sedentary behaviour in Chile: results from the National Health Survey 2009-2010. J Public Health (Oxford, England) 2016; 38 (2): e98-e105.

25. MINSAL. Encuesta Nacional de Salud 2016-2017. Ministerio de Salud, Chile. 2017; Available: http://www. minsal.cl/wp-content/uploads/2017/11/ENS-201617_PRIMEROS-RESULTADOS.pdf.

26. Mehta N, Myrskyla M. The Population Health Benefits Of A Healthy Lifestyle: Life Expectancy Increased And Onset Of Disability Delayed. Health Aff (Project Hope). 2017.

27. Zhang X, Devlin HM, Smith B, Imperatore G, Thomas W, Lobelo F, et al. Effect of lifestyle interventions on cardiovascular risk factors among adults without impaired glucose tolerance or diabetes: A systematic review and meta-analysis. PloS One 2017; 12 (5): e0176436.

28. VanWormer JJ, Boucher JL, Sidebottom AC, Sillah A, Knickelbine T. Lifestyle changes and prevention of metabolic syndrome in the Heart of New Ulm Project. Prev Med Rep 2017; 6: 242-5.

29. van Dammen L, Wekker V, van Oers AM, Mutsaerts MAQ, Painter RC, Zwinderman AH, et al. Effect of a lifestyle intervention in obese infertile women on cardiometabolic health and quality of life: A randomized controlled trial. PloS One 2018; 13 (1): e0190662. 
30. Bakrania K, Edwardson CL, Bodicoat DH, Esliger DW, Gill JM, Kazi A, et al. Associations of mutually exclusive categories of physical activity and sedentary time with markers of cardiometabolic health in English adults: a cross-sectional analysis of the Health Survey for England. BMC Public Health 2016; 16: 25.

31. Sotos-Prieto M, Bhupathiraju SN, Falcon LM, Gao X, Tucker KL, Mattei J. A Healthy Lifestyle Score Is Associated with Cardiometabolic and Neuroendocrine Risk Factors among Puerto Rican Adults. J Nutr 2015; 145 (7): 1531-40.

32. Del Gobbo LC, Kalantarian S, Imamura F, Lemaitre R, Siscovick DS, Psaty BM, et al. Contribution of Major Lifestyle Risk Factors for Incident Heart Failure in Older Adults: The Cardiovascular Health Study. JACC Heart Fail 2015; 3 (7): 520-8.

33. Chen C-C, Liu K, Hsu C-C, Chang H-Y, Chung H-C, Liu J-S, et al. Healthy lifestyle and normal waist circumference are associated with a lower 5-year risk of type 2 diabetes in middle-aged and elderly individuals: Results from the healthy aging longitudinal study in Taiwan (HALST). Medicine 2017; 96 (6): e6025.

34. Liu K, Daviglus ML, Loria CM, Colangelo LA, Spring B, Moller AC, et al. Healthy lifestyle through young adulthood and the presence of low cardiovascular disease risk profile in middle age: the Coronary Artery Risk Development in (Young) Adults (CARDIA) study. Circulation 2012; 125 (8): 996-1004.

35. Celis-Morales CA, Pérez-Bravo F, Ibáñez L, Salas C, Bailey ME, Gill JM. Objective vs self-reported physical activity and sedentary time: effects of measurement method on relationships with risk biomarkers. PLoS One. 2012; 7 (5): e36345.

36. Celis-Morales C, Livingstone KM, Woolhead C, Forster $\mathrm{H}$, O’Donovan CB, Macready AL, et al. How reliable is internet-based self-reported identity, socio-demographic and obesity measures in European adults? Genes Nutr 2015; 10(5): 28. 